VASA/CR-GD- 207115

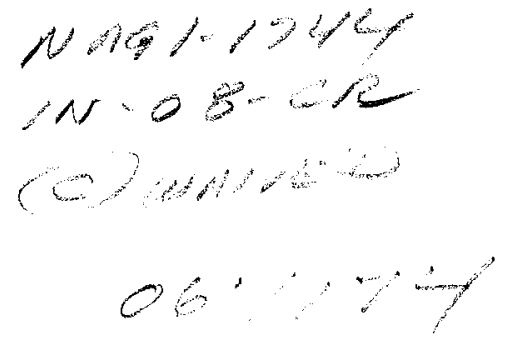

\title{
Unified Theory for Aircraft Handling Qualities and Adverse Aircraft-Pilot Coupling R. A. Hess
}

Reprinted from

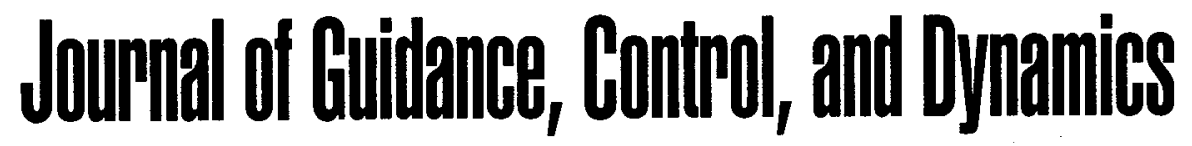

Volume 20 , Number 6 , Pages $1141-1148$

\section{OAfa}

A publication of the

American Institute of Aeronautics and Astronautics, Inc.

1801 Alexander Bell Drive, Suite 500

Reston, VA 20191-4344 


\title{
Unified Theory for Aircraft Handling Qualities and Adverse Aircraft-Pilot Coupling
}

\author{
R. A. Hess* \\ University of California, Davis, Davis, California 95616
}

\begin{abstract}
A unified theory for aircraft handling qualities and adverse aircraft-pilot coupling or pilot-induced oscillations is introduced. The theory is based on a structural model of the human pilot. A methodology is presented for the prediction of 1) handling qualities levels, 2) pilot-induced oscillation rating levels, and 3) a frequency range in which pilot-induced oscillations are likely to occur. Although the dynamics of the force-feel system of the cockpit inceptor is included, the methodology will not account for effects attributable to control sensitivity and is limited to single-axis tasks and, at present, to linear vehicle models. The theory is derived from the feedback topology of the structural model and an examination of flight test results for 32 aircraft configurations simulated by the U.S. Air Force/CALSPAN NT-33A and Total In-Flight Simulator variable stability aircraft. An extension to nonlinear vehicle dynamics such as that encountered with actuator saturation is discussed.
\end{abstract}

\section{Introduction}

A $\mathrm{N}$ adverse aircraft-pilot coupling (APC) or pilot-induced oscillation (PIO) can be defined as an unwanted, inadvertent, and atypical closed-loop coupling between a pilot and the response variables of an aircraft. ${ }^{1}$ APC or PIO problems are not new phenomena; indeed, they have been around since the Wright Brothers and have been referred to as the senior handling qualities problem. ${ }^{2}$ McRuer $^{2}$ gives a concise historical perspective of the PIO problem, including a review and discussion of germane pilot behavior patterns.

Because of a strong correlation between APC/PIO susceptibility and modern, full-authority control systems employing fly-by-wire (FBW) technology, interest in studying the APC/PIO phenomenon has been increasing. For example, NATO's AGARD convened a special workshop on PIO, ${ }^{3}$ and NASA has sponsored a National Research Council committee to study the problem of APC/PIO. ${ }^{4}$ U.S. Air Force interest in the APC/PIO problem has led to the publication of four reports under the general rubric of a Unified PIO Study. ${ }^{5-8}$

Despite the amount of research that has been directed toward solution of the APC/PIO problem, there appears to be little consensus about the phenomenon itself in terms of the pilot behavior that initiates and sustains the APC/PIO. There is general agreement that the contributing factors are 1) a demanding flight task, 2) a vehicle with unsatisfactory dynamics, and 3 ) a triggering event. ${ }^{2}$ The main thrust of the research to be described is to suggest one possible humancentered theory concerning the APC/PIO phenomenon. The theory to be proposed will attempt to unify the topics of vehicle handling qualities and APC/PIO, which have been somewhat disparate in the past. Finally, although pertinent to the handling qualities of highperformance aircraft, the phenomenon of roll ratchet ${ }^{9}$ will not be discussed here.

\section{Revised Structural Model}

Figure 1 shows what will be referred to here as the revised structural model of the human pilot. The model has its genesis in a previously described structural model ${ }^{10}$ and in a later modification of that model. ${ }^{11}$ As shown in Fig. 1, the model is describing compensatory pilot behavior, i.e., behavior involving closedloop tracking in which the visual input is system error. The elements within the dashed box represent the dynamics of the human pilot.

\footnotetext{
Received Oct. 11, 1996; presented as Paper 97-0454 at the AIAA 35th Aerospace Sciences Meeting, Reno, NV, Jan. 6-9, 1997; revision received March 20,1997; accepted for publication March 21, 1997. Copyright (C) 1997 by R. A. Hess. Published by the American Institute of Aeronautics and Astronautics, Inc., with permission.

${ }^{*}$ Professor, Department of Mechanical and Aeronautical Engineering. Associate Fellow AIAA.
}

Starting from the left, one sees the system error $e(t)$ following one of two possible paths. One path is intended to model the human's visual rate-sensing dynamics, here modeled by a differentiator $(s)$, an injected noise signal, and a gain $K_{\dot{\varepsilon}}$. The remaining path describes normal error sensing and gain compensation $K_{e}$, including the possibility of the human's accomplishing low-frequency trim (or integral) compensation via $\epsilon / s$. In the study herein, $\epsilon \equiv 0$. The switch labeled $S_{1}$ allows switching between error and errorrate tracking. This switching will be hypothesized to play a critical role in the initiation and sustenance of APC/PIOs. A central processing time delay $\tau_{0}$ is also included. An inner, proprioceptive feedback loop is encountered next. Proprioceptive feedback implies the use of sensory information about limb position. In the forward portion of this loop, the elements $Y_{\mathrm{NM}}$ and $Y_{\mathrm{FS}}$ are intended to represent, respectively, the open-loop dynamics of the neuromuscular system driving the cockpit inceptor and the dynamics of the inceptor force-feel system itself. The feedback portion of this loop contains the element $Y_{\mathrm{PF}}$, which receives as its input the proprioceptively sensed inceptor output $\delta_{m}(t)$. The element $Y_{\mathrm{PF}}$ and its position in the model are central to the philosophy of the structural model, i.e., that the primary equalization capabilities of the human pilot are assumed to occur through operation upon a proprioceptively sensed, as opposed to a visually sensed, variable. As will be discussed, switches $S_{1}$ and $S_{2}$ are assumed to operate in unison, i.e., when $S_{1}$ is in the "up" position, so is $S_{2}$. Unless stated otherwise, it is assumed in what follows that switches $S_{1}$ and $S_{2}$ are in the nominal, "down" position. The switch $S_{3}$ allows either displacement-sensing or force-sensing inceptors to be modeled.

Time derivatives of the vehicle output $m(t)$ are assumed to be individually sensed, as indicated in Fig. 1 . Switch $S_{4}$ allows either rate or acceleration cues or neither to be used in vehicular control. Note that feeding back output rate is predicated on that signal creating an acceleration that can be sensed by the middle ear. In this study, $K_{\bar{m}}=K_{\dot{m}}=0$. A visual feedback of vehicle output completes the model.

The particular form of the error-rate loop deserves some comment. It is the author's contention that the visual sensing of rate information of a quality suitable for precise closed-loop tracking is compromised by the limitations of the human visual system. The injected noise has been included as a somewhat crude model of these limitations. The noise itself is not pertinent to the discussion and will not be treated further.

\section{Pilot-Vehicle Analysis with the Structural Model}

Model Parameterization

As can be seen from Fig. 1, only the elements $Y_{\mathrm{NM}}$ and $Y_{\mathrm{PF}}$ need to be parameterized because the remaining elements are simply gains, 


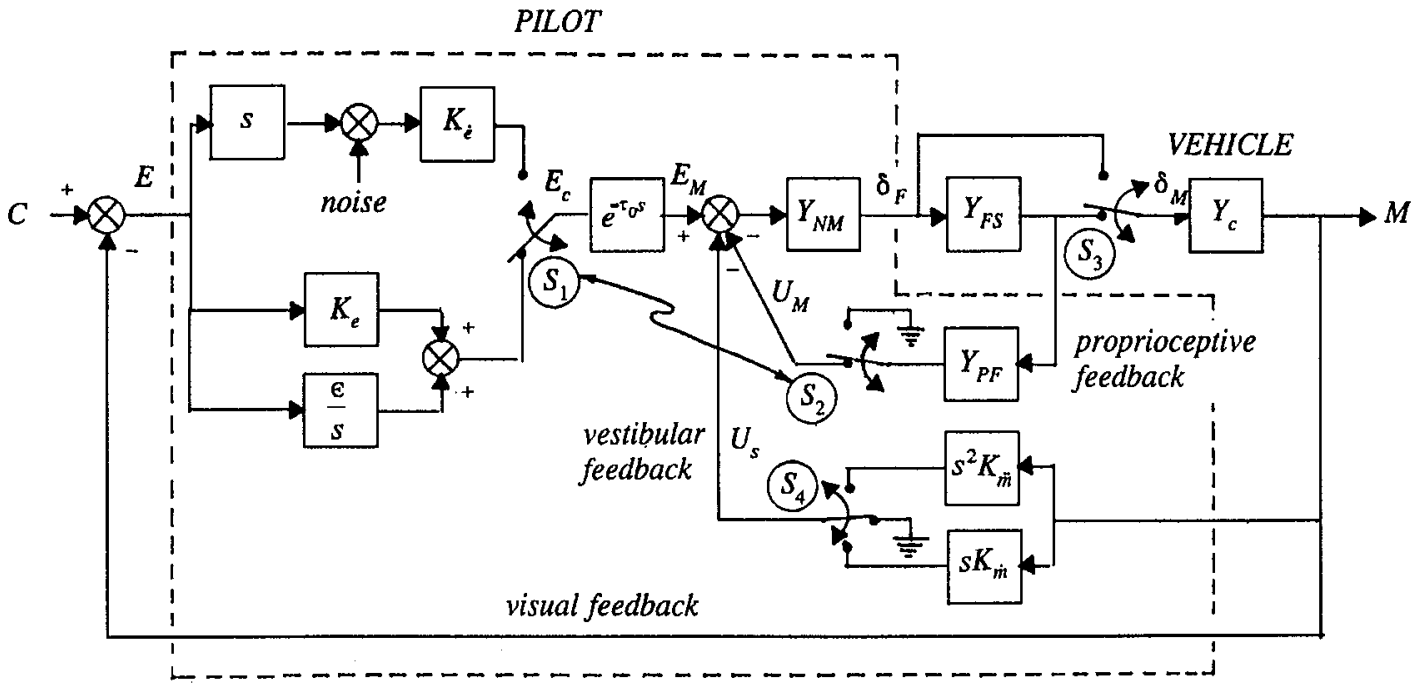

Fig. 1 Revised structural model of the human pilot.

e.g., $K_{\dot{e}}, K_{e}$, etc., differentiating elements $\left(s, s^{2}\right)$, and time delays $\left(\tau_{0}\right)$. Here,

$$
\begin{gathered}
Y_{\mathrm{NM}}=\frac{\omega_{\mathrm{NM}}^{2}}{s^{2}+2 \zeta_{\mathrm{NM}} \omega_{\mathrm{NM}} s+\omega_{\mathrm{NM}}^{2}} \\
Y_{\mathrm{PF}}=K(s+a) \quad \text { or } \quad K \quad \text { or } K /(s+a)
\end{gathered}
$$

with the particular equalization of Eqs. (2) dependent on the form of the vehicle dynamics around the crossover frequency.

The forms of Eqs. (2) can be interpreted as the pilot's "internal model" of the vehicle dynamics. That is, in the range of crossover, $Y_{\mathrm{PF}} \propto s \cdot Y_{c}(s)$. There will be reason to return to this internal model concept in discussing pilot behavior in APC/PIO events later.

\section{Crossover Frequency Selection}

In the analyses to follow, a constant crossover frequency $\omega_{c}=$ $2.0 \mathrm{rad} / \mathrm{s}$ is chosen. Of course, the human pilot can, in a limited manner, vary crossover frequency. ${ }^{12}$ However, for the purposes of analysis, the assumption of a constant crossover frequency is very useful. Selecting $\omega_{c}=2.0 \mathrm{rad} / \mathrm{s}$ is not an arbitrary decision. Using the crossover model of the human pilot, ${ }^{12}$ Ref. 13 examined the sensitivity of the closed-loop system bandwidth $\omega_{B}$ to changes in the open-loop crossover frequency. It was shown that, for $\omega_{c}<$ $0.34 / \tau_{e}$ (where $\tau_{e}$ is the crossover model's effective time delay), $\omega_{B}$ becomes very sensitive to reductions in $\omega_{c}$. Selecting a value for $\tau_{e}$ representative of the lower limit found in the literature $\left(\tau_{e}=0.2 \mathrm{~s}\right)$ leads to a "critical" value of $\omega_{c}=1.7 \mathrm{rad} / \mathrm{s}$. This value was rounded up to $2.0 \mathrm{rad} / \mathrm{s}$ for the purposes of this study.

\section{Parameter Value Selection}

As in applications of the original structural model, a number of model parameters will be considered invariant across different vehicles and tasks. The particular values chosen are considered representative of values associated with the dynamic subsystem being modeled. Choosing the undamped natural frequency of the openloop neuromuscular system as $\omega_{\mathrm{NM}}=10 \mathrm{rad} / \mathrm{s}$ is one such example. Nominal values for these "fixed" parameters can be given as

$$
\tau_{0}=0.2 \mathrm{~s}, \quad \omega_{\mathrm{NM}}=10 \mathrm{rad} / \mathrm{s}, \quad \zeta_{\mathrm{NM}}=0.7
$$

The relatively simple relations of Eqs. (1-3), the crossover relation $\omega_{c}=2.0 \mathrm{rad} / \mathrm{s}$, and the selection of one of the three forms on the right-hand side of Eqs. (2) allow implementation of the model of Fig. 1. The appropriate form in Eqs. (2) is chosen so that the resulting open-loop transfer function

$$
Y_{p} Y_{c}(j \omega)=\left(\delta_{M} / E\right)(j \omega) \cdot Y_{c}(j \omega) \approx\left(\omega_{c} / j \omega\right) e^{-\tau_{e} \cdot s}
$$

i.e., $Y_{p} Y_{c}(j \omega)$ follows the dictates of the crossover model of the human pilot. ${ }^{12}$ The implementation of Eq. (4) is critical to the success of the handling qualities and APC/PIO analyses to follow, so it is important to specify in a precise manner just how this is done. Limiting discussion to the last two forms of $Y_{\mathrm{PF}}$ (those most likely to be encountered in pilot-vehicle analyses), the right-hand side of Eqs. (2) is selected so that

$$
\frac{Y_{c}(j \omega)}{Y_{\mathrm{PF}}(j \omega)} \approx \frac{K_{1}}{j \omega} \quad \text { for } \quad\left\{\begin{array}{l}
\omega \approx \omega_{c} \\
K_{1} \text { arbitrary }
\end{array}\right.
$$

The gain $K$ appearing in Eqs. (2) is chosen so that, with all other loops open, the minimum damping ratio of any quadratic closedloop poles of $\left(\delta_{M} / E_{M}\right)(s)$ is $\zeta_{\min }=0.15$. Finally, $K_{e}$ is selected so that the desired crossover frequency of $2.0 \mathrm{rad} / \mathrm{s}$ is obtained.

The use of fixed parameters in the pilot model is obviously an analytical simplification. However, it has been the author's experience that the model so derived is of sufficient accuracy to justify its use in the pilot-vehicle analyses that are the subject of the research to be described. As described, the model will reflect the important, experimentally verified human pilot dynamics, ${ }^{12}$ i.e., faithful reproduction of crossover model characteristics across different vehicles and the important dynamics of the closed-loop neuromuscular system, including high-frequency amplitude peaking and phase roll-off.

\section{Previous Results}

\section{Analysis of Handling Qualities}

A theory for handling qualities based on the original structural model has been proposed and discussed elsewhere. ${ }^{12,14}$ The theory postulates that the power in the proprioceptive feedback signal $u_{m}(t)$ of Fig. 1 is the determining factor in a pilot's perception of a vehicle's handling qualities when Eq. (4) is satisfied. The signal $u_{m}(t)$ can be shown to be proportional to the output rate $\dot{m}(t)$ due to control activity $\delta_{m}(t)$. Because the power in $u_{m}(t)$ is dependent on $\left|\left(U_{M} / C\right)(j \omega)\right|$, it was found that this function itself could be used to predict handling qualities levels and was referred to as the handling qualities sensitivity function (HQSF). ${ }^{12,14}$ The similarity between the previous structural model and its revised incarnation in Fig. 1 allows the HQSF definition to remain unchanged:

$$
\mathrm{HQSF}=\left|\left(U_{M} / C\right)(j \omega)\right|
$$

In calculating the HQSF, it is necessary to remove the effects of control sensitivity. By this it is meant that the model results are forced to be independent of control and force-feel system sensitivity. This sensitivity includes command path gains between the inceptor and the actuators and the static gain of the pertinent vehicle transfer function, i.e., the gain appearing in the vehicle transfer function when written in "time-constant" form. Removing the effects of uncertainty in the HQSF is accomplished as follows: 
Displacement-sensing inceptor

$$
\mathrm{HQSF}=\left|\frac{M}{C}(j \omega) \cdot \frac{1}{K_{e}} \cdot \frac{1}{Y_{c}(j \omega)} \cdot Y_{\mathrm{PF}}(j \omega)\right|
$$

Force-sensing inceptor

$$
\mathrm{HQSF}=\left|\frac{M}{C}(j \omega) \cdot \frac{1}{K_{e}} \cdot \frac{1}{Y_{c}(j \omega)} \cdot Y_{\mathrm{FS}} Y_{\mathrm{PF}}(j \omega)\right|
$$

For level 1 handling qualities, i.e., $1 \leq$ Cooper-Harper ratings $\leq$ 3.5 , the previous structural model required $\mathrm{HQSF}<1.0$. However, because the previous model differed in detail, especially in the proprioceptive feedback loop, the criteria for level 1 handling qualities will probably change when the model of Fig. 1 is used. In addition, there were no bounds suggested for level 2 and 3 handling quality levels in previous handling quality studies with the original structural model. This restriction will be removed in what follows.

\section{New Results}

Flight-test handling quality results from Refs. 15-18 were used to obtain new bounds for the HQSFs. The data concentrated on the longitudinal approach and landing tasks for the U.S. Air Force/CALSPAN NT-33A ${ }^{15,16}$ and Total In-Flight Simulator (TIFS) $^{17,18}$ vehicles. The configurations shown in Table 1 were selected to give as wide a distribution in handling qualities and APC/PIO susceptibility as possible. Because the pilot-vehicle analysis technique does not consider the effects of control sensitivity, the question of how the flight-test control sensitivities were determined naturally arises. The "back seat" NT-33A pilot selected what he determined to be the optimum sensitivity for each configuration in Ref. 15. This value remained unchanged for the other evaluation pilots. In Ref. 16, each evaluation pilot determined what he believed to be the optimum sensitivity for each configuration. In Refs. 17 and 18 , an attempt was made to achieve comparable sensitivities across all configurations by selecting the control sensitivity that produced the same maximum pitch rate for a step inceptor input. One cannot guarantee that these procedures eliminated control sensitivity effects from the handling qualities and APC/PIO susceptibility, but their impact was minimized.

Given the necessary information on vehicle and force-feel system dynamics, pilot-vehicle analyses were undertaken as just described for the 32 configurations identified in Table 1 . The average CooperHarper Pilot Opinion Ratings (PORs) that these configurations received in flight tests are also given. In cases in which separate overall and approach/flare/landing ratings were elicited, just the averaged overall ratings were used.

In undertaking the pilot-vehicle analyses, two exceptions to the $2.0-\mathrm{rad} / \mathrm{s}$ crossover frequency rule were necessary. These exceptions occurred with configuration 5-3 (Ref. 15) and configuration H2-8 (Ref. 16). In each case, an attempt to apply Eq. (5) resulted in the open-loop pilot-vehicle transfer function exhibiting a flat amplitude region about crossover. Thus, small changes in pilot gain resulted in large changes in stability margins that the author did not consider to be realistic pilot-vehicle characteristics. The following procedure was implemented to handle these cases. First, an attempt was made to increase the crossover frequency until the offending flat portion of the amplitude plot was at least $2.0 \mathrm{~dB}$ above the $0-\mathrm{dB}$ line. If this change could not be accomplished with positive stability margins, the crossover frequency was reduced to $1 \mathrm{rad} / \mathrm{s}$ for that configuration. The latter approach was necessary for configuration 5-3, whereas the former sufficed for configuration $\mathrm{H} 2-8$.

Figure 2 shows the HQSFs resulting from the pilot-vehicle analyses of the configurations that received level 1 PORs in flight tests. The dashed line approximates a least upper bound for these HQSFs. Figure 3 shows a similar plot for the configurations that received level 2 PORs. The upper dashed line again provides an approximate upper bound. Finally, Fig. 4 does the same for configurations that received level 3 PORs. (For the purposes of this research, level 3 was considered to include all PORs $>6.5$.) The only "failure" in this categorization of $32 \mathrm{HQSFs}$ is that for configuration 4 in the

\begin{tabular}{|c|c|c|c|c|c|c|c|c|c|c|c|}
\hline \multicolumn{3}{|c|}{ Ref. 15} & \multicolumn{3}{|c|}{ Ref. 16} & \multicolumn{3}{|c|}{ Ref. 17} & \multicolumn{3}{|c|}{ Ref. 18} \\
\hline Configuration & POR & PIOR & Configuration & POR & PIOR & Configuration & POR & $\overline{\text { PIOR }}$ & Configuration & POR & PIOR \\
\hline $1-3$ & 9.5 & 3.25 & $\mathrm{H} 2-1$ & 2.33 & 1.0 & B & 2.7 & 1.0 & $5-2-2$ & 2.5 & 1.0 \\
\hline $1-\mathrm{C}$ & 4.0 & 1.0 & $\mathrm{H} 2-5$ & 9.0 & 4.33 & 1 & 2.9 & 1.0 & $6-1-1$ & 5.0 & 2.5 \\
\hline $2-\mathrm{C}$ & 2.5 & 1.0 & $\mathrm{H} 2-8$ & 8.67 & 4.0 & 4 & 3.75 & 1.25 & & & \\
\hline $3-\mathrm{C}$ & 5.0 & 1.25 & H3-1 & 4.0 & 2.33 & 11 & 5.7 & 3.33 & & & \\
\hline $4-4$ & 6.5 & 2.67 & $\mathrm{H} 3-3$ & $2.5^{\mathrm{a}}$ & 1.66 & 17 & 2.6 & 1.0 & & & \\
\hline $4-7$ & 3.0 & 1.0 & H3-6 & 4.5 & 2.0 & $17 \mathrm{~L}+\mathrm{L}$ & 3.5 & 1.0 & & & \\
\hline $4-10$ & 9.0 & 4.0 & H3-D & 2.0 & 1.0 & 20 & 3.5 & 1.0 & & & \\
\hline $5-3$ & 6.1 & 2.1 & H3-12 & 8.0 & 4.5 & 21 & 4.5 & 2.25 & & & \\
\hline $6-1$ & 10.0 & 4.0 & $\mathrm{H} 4-1$ & 2.67 & 1.0 & 22 & 6.75 & 4.0 & & & \\
\hline $6-2$ & 2.0 & 1.0 & H5-10 & 10.0 & 5.0 & & & & & & \\
\hline & & & H5-11 & 6.33 & 3.0 & & & & & & \\
\hline
\end{tabular}

Table 1 Configurations for handling qualities and APC/PIO investigation

aThee ratings of $7 / 2 / 3$ were given. Reference 16 lists this as level 1 . The author does the same and assumes that the pilot rating of 7.0 was treated as anomalous.

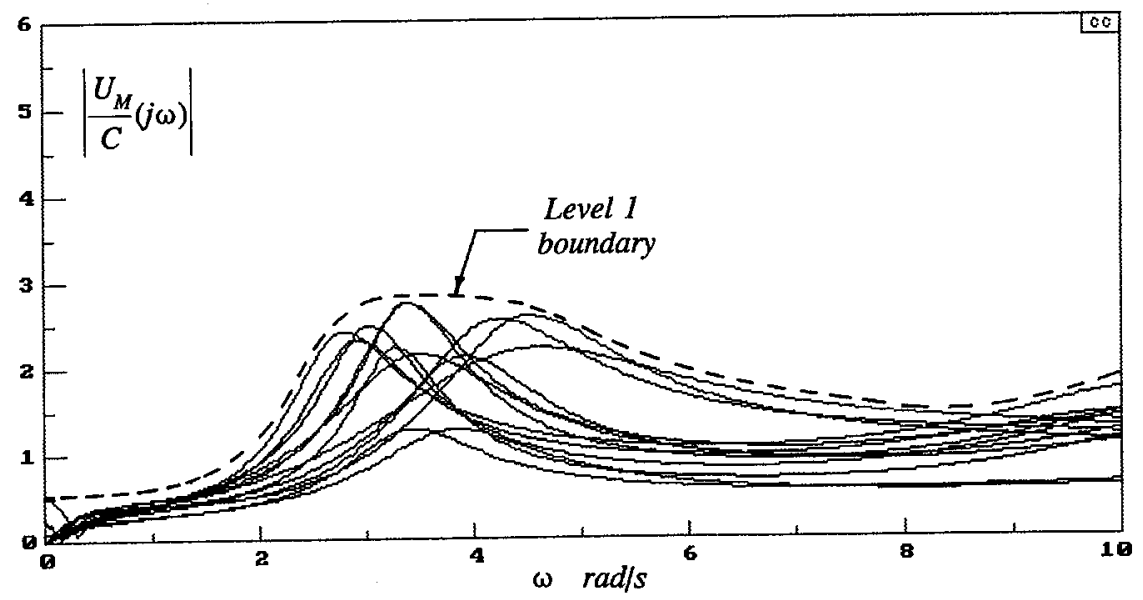

Fig. 2 HQSFs for flight-test configurations receiving level 1 PORs. 


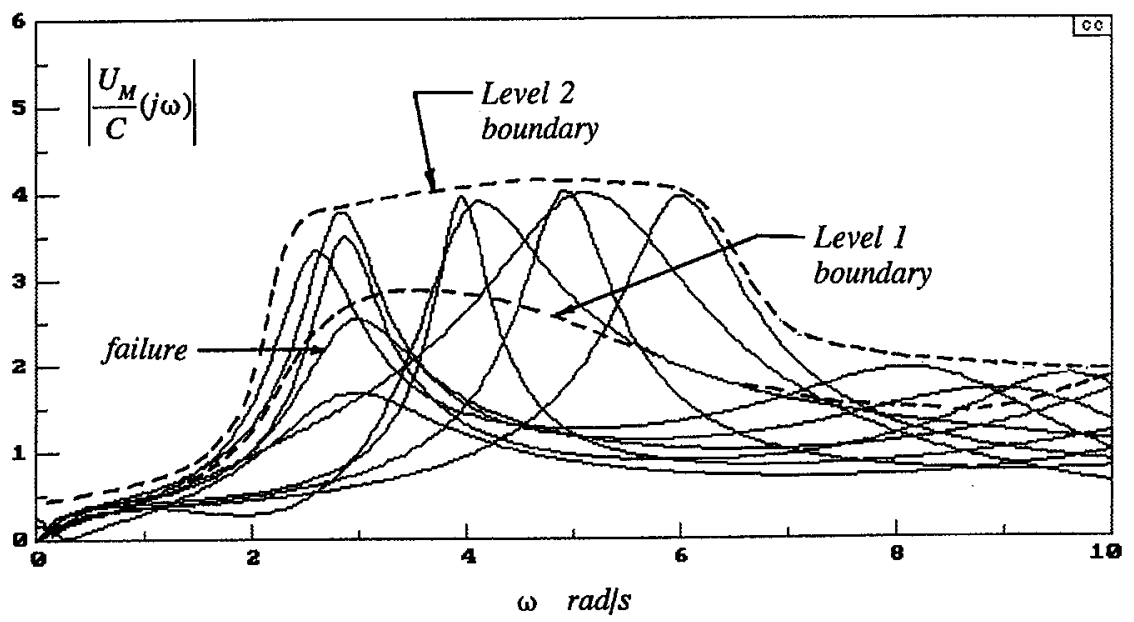

Fig. 3 HQSFs for flight-test configurations receiving level 2 PORs.

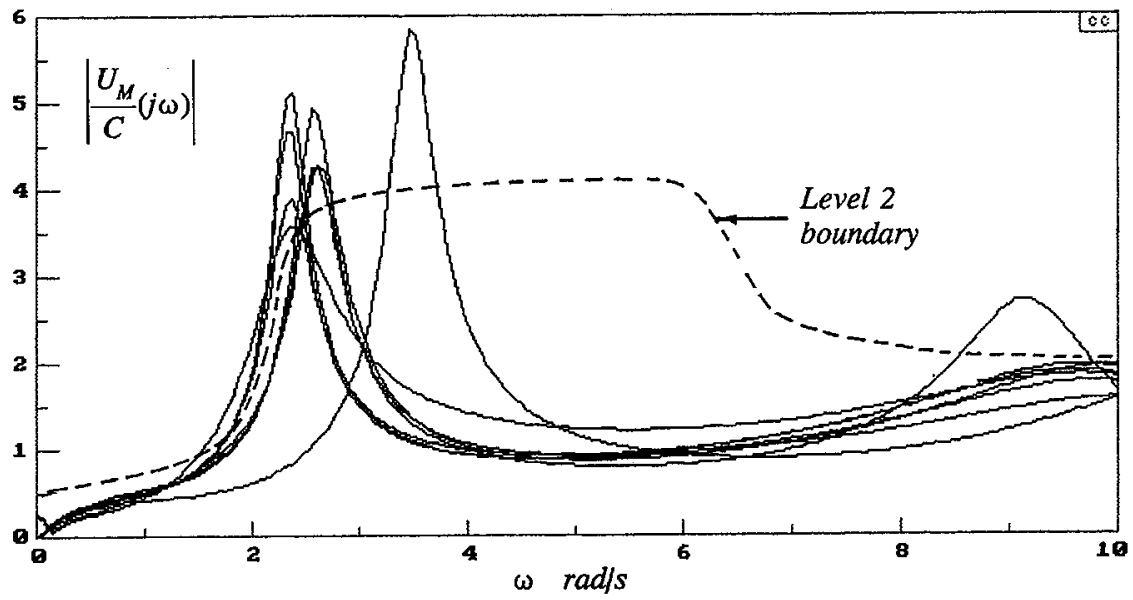

Fig. 4 HQSFs for flight-test configurations receiving level 3 PORs.

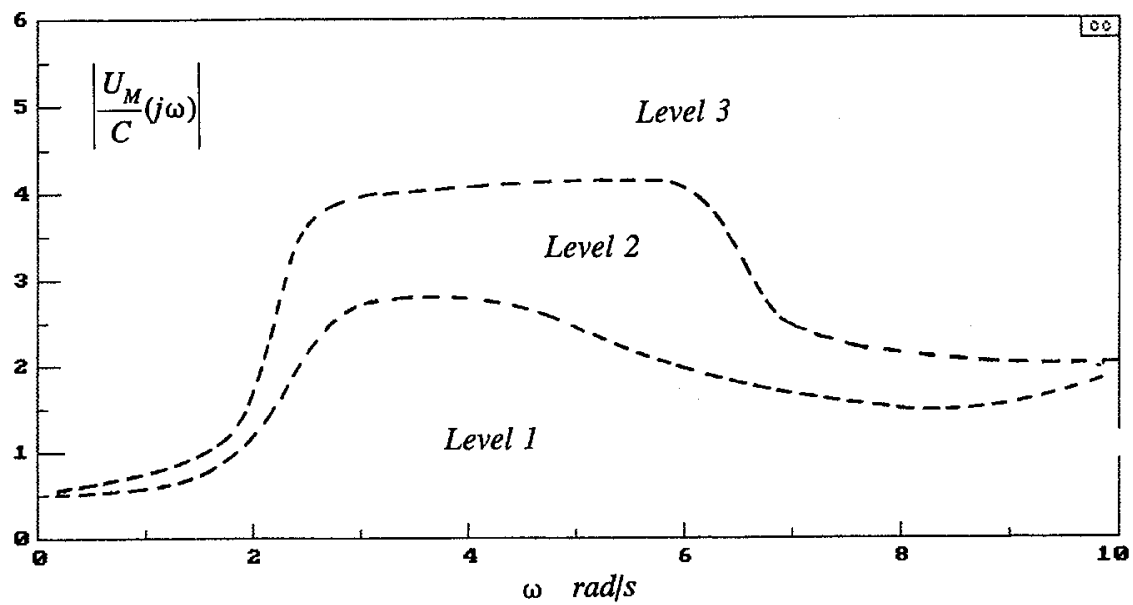

Fig. 5 Handling quality boundaries for HQSFs.

TIFS data of Ref. 17. Figure 3 would place this configuration in level 1 , whereas the average POR reported in flight tests was 3.75 , which would place this configuration just within level 2 .

Figure 5 summarizes all of the handling quality boundaries. Figure 5 implies that, if the HQSF resulting from application of the pilot-vehicle analysis described in the preceding is below the level 1 boundary, the configuration should be expected to receive level 1 PORs. If the HQSF exceeds the level 1 boundary but remains below the level 2 boundary, the configuration should be expected to receive level 2 PORs. Finally, if the HQSF exceeds the level 2 boundary, it should be expected to receive level 3 PORs. The theoretical basis for this categorization derives from the hypothesized importance of the signal $u_{m}(t)$ in the pilot model.

\section{Analysis of APC/PIO Events}

PIO Ratings

An analysis of the vehicle configurations of Table 1 using the pilot-vehicle analysis procedure described in the preceding was conducted with the goal of developing a theory for APC/PIO. Again, the characteristics of the proprioceptive feedback signal $u_{m}(t)$ were investigated in this context. It was found that a sensitive metric for APC/PIO susceptibility was the power spectral density (PSD) of the signal $u_{m}(t)$ when a filtered white noise command $c(t)$ was applied. The PSD of $c(t)$ was selected as

$$
\Phi_{c c}(\omega)=\frac{4^{2}}{\omega^{4}+4^{2}}
$$


Table 2 PIOR scale description

\begin{tabular}{lc}
\hline \hline Description & Numerical rating \\
\hline $\begin{array}{l}\text { No tendency for pilot to induce undesirable motions. } \\
\text { Undesirable motions tend to occur when pilot initiates abrupt maneuvers or attempts } \\
\text { tight control. These motions can be prevented or eliminated by pilot technique. } \\
\text { Undesirable motions easily induced when pilot initiates abrupt maneuvers or attempts tight } \\
\text { control. These motions can be prevented or eliminated but only at sacrifice to task performance } \\
\text { or through considerable pilot attention and effort. }\end{array}$ & 2 \\
$\begin{array}{l}\text { Oscillations tend to develop when pilot initiates abrupt maneuvers or attempts tight control. } \\
\text { Pilot must reduce gain or abandon task to recover. }\end{array}$ & 3 \\
$\begin{array}{l}\text { Divergent oscillations tend to develop when pilot initiates abrupt maneuvers or attempts } \\
\text { tight control. Pilot must open loop by releasing or freezing the stick. } \\
\text { Disturbance or normal pilot control may cause divergent oscillation. Pilot must open control } \\
\text { loop by releasing or freezing the stick. }\end{array}$ & 4 \\
\hline
\end{tabular}

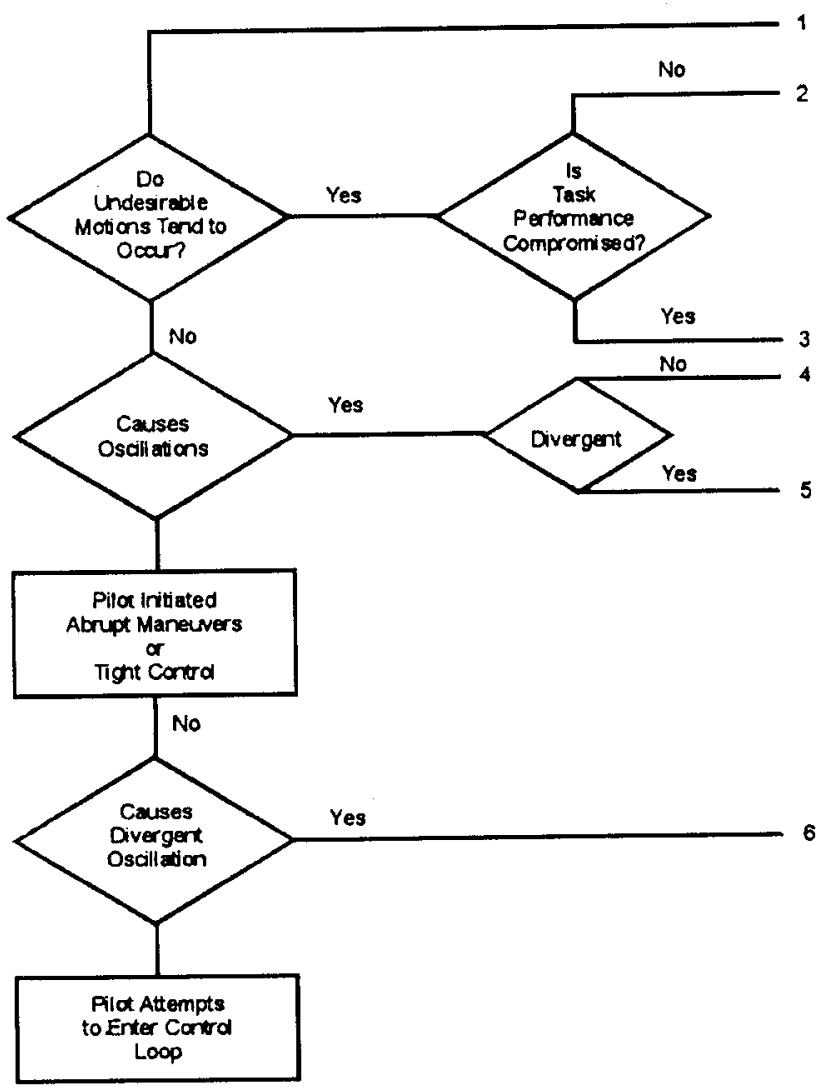

Fig. 6 PIOR scale.

$\Phi_{c c}(\omega)$ was chosen with a break frequency at $2.0 \mathrm{rad} / \mathrm{s}$, identical to the crossover frequency enforced in the pilot-vehicle analysis. It was found that, similar to the HQSF, plots of $\Phi_{u_{m} u_{n}}(\omega)$ could be used to delineate "levels" of pilot-induced oscillation ratings (PIORs) using the scale of Fig. 6 and Table 2. The levels were defined herein as

$$
1 \leq \mathrm{PIOR} \leq 2, \quad 2<\mathrm{PIOR}<4, \quad \text { PIOR } \geq 4
$$

Table 1 lists the averaged PIORs that each of the configurations received in flight test. Figure 7 shows $\Phi_{u_{m} u_{m}}(\omega)$ [defined as $\Phi_{c c}(\omega)$. $\left.[\mathrm{HQSF}]^{2}\right]$ for the configurations that received $1 \leq \mathrm{PIOR} \leq 2$. Figure 8 shows $\Phi_{u_{m} u_{m}}(\omega)$ for configurations that received $2<$ PIOR $<4$, and finally, Fig. 9 shows $\Phi_{u_{m} u_{m}}(\omega)$ for configurations that received PIOR $\geq 4$. In each of these figures, the dashed lines approximate a least upper bound for the PSDs in question. The nature of the PSDs and their relation to the PIORs has allowed simpler bounds to be drawn than those of Fig. 5. Also note that, as opposed to Figs. 2-5, the ordinates in Figs. 7-9 have different scales. There is one failure in this categorization of the PIORs. In Fig. 8, configuration H3-1 (Ref. 16) lies in the area indicating $1 \leq$ PIOR $<2$, whereas Table 1 indicates that it received an average PIOR of 2.33 from flight tests.

As in the case of the HQSF boundaries of Fig. 5, Figs. 7-9 suggest that one can delineate between the PIOR levels of Eq. (9) using
$\Phi_{u_{n} u_{m}}(\omega)$. Figure 9 implies that, if the $\Phi_{u_{n n} u_{m}}(\omega)$ resulting from application of the pilot-vehicle analysis described in the preceding is below the lowest bound, the configurations should be expected to receive a $1 \leq$ PIOR $\leq 2$. If the $\Phi_{u_{m} u_{m}}(\omega)$ exceeds the lowest bound in Fig. 9 but remains below the next bound, the configuration should be expected to receive a $2<$ PIOR $<4$. Finally, if the $\Phi_{u_{n} u_{n}}(\omega)$ exceeds the upper bound in Fig. 9 , the configuration should be expected to receive a PIOR $\geq 4$. The theoretical basis for this categorization is again based on the hypothesized importance of the signal $u_{m}(t)$.

\section{Development of an APC/PIO Event}

As mentioned in a preceding section, conditions almost invariably accompanying an APC/PIO event are 1) a demanding flight task, 2) a vehicle with unsatisfactory dynamics, and 3 ) a triggering event. In terms of the pilot-vehicle analysis technique that has been described, the first two conditions are respectively realized by 1) the existence of a relatively high crossover frequency in a compensatory tracking pilot model and 2) the evidence of $\Phi_{u_{m} u_{m}}(\omega)$ exceeding the boundary associated with PIOR $\geq 4$. The triggering event itself is not part of the pilot-vehicle model. Triggers can originate in the external environment, e.g., a sudden patch of turbulence, the vehicle, e.g., a stability augmentation transient, or the pilot him/herself, e.g., a change in the vehicle response variable being actively controlled. However, the effect of this event is hypothesized to cause switches $S_{1}$ and $S_{2}$ in Fig. 1 to move to the position in which the pilot tracks error rate instead of error and does so without proprioceptive feedback. It is hypothesized that this altered feedback structure represents the pilot's temporary regression to a type of tracking behavior that can occur in initial exposure to a new dynamic system. In the latter case, no internal model has been formed through training (no $Y_{\mathrm{PF}}$ to permit proprioceptive feedback and adequate compensation of the plant), and the task becomes one of simply keeping the error bounded by attempting to control error rate with no compensation. Obviously, in an operational setting, this behavior is unwanted, inadvertent, and atypical.

\section{APC/PIO Frequency}

The legitimacy of any analytical pilot-vehicle model that is used to study APC/PIO events is often judged by the ability of that model to predict APC/PIO frequencies that have been measured in experiment. Because the model of Fig. 1 is linear, conditions of neutral stability (with switches $S_{1}$ and $S_{2}$ in the up position indicating rate tracking with no proprioceptive feedback) can be easily obtained. This includes the unstable frequency (frequency of oscillation). Define the loop transmission with rate tracking as $L_{r}$, where from Fig. 1,

$$
L_{r}=\left.s \cdot K_{\dot{e}} \cdot\left(M / E_{C}\right)\right|_{S_{1}, S_{2} \text { up position }}
$$

Neutral stability will occur when

$$
\angle L_{r}(j \omega)=-180 \mathrm{deg}
$$

Configuration 22 from Ref. 17 can be selected as an example. As Table 1 indicates, the configuration received an average 


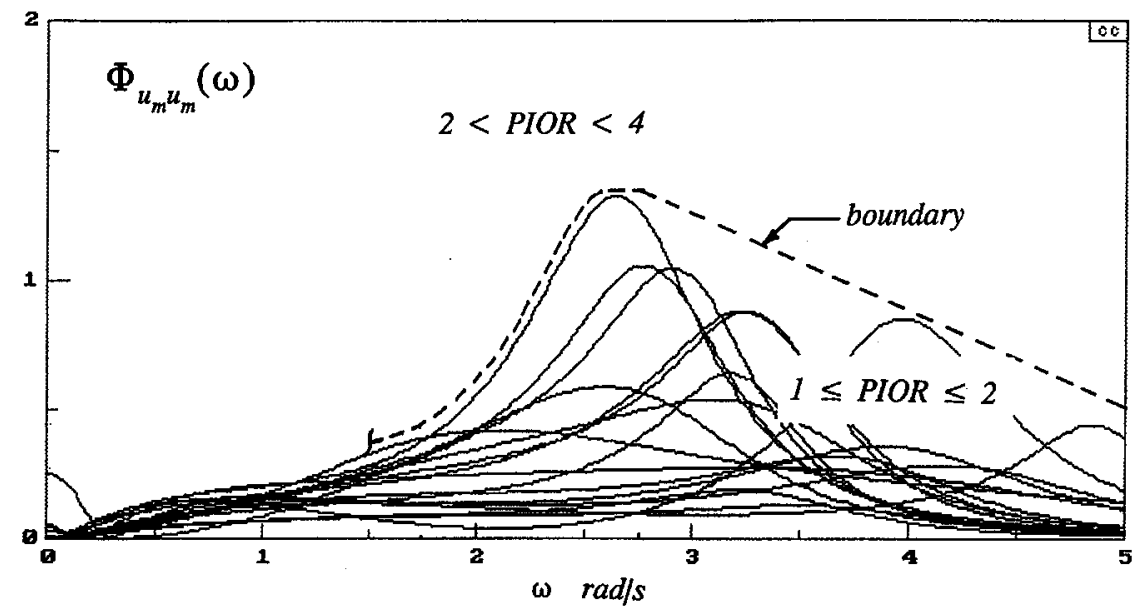

Fig. $7 \Phi_{u_{m} u_{m}}(\omega)$ for flight-test configurations receiving $1 \leq$ PIOR $\leq 2$.

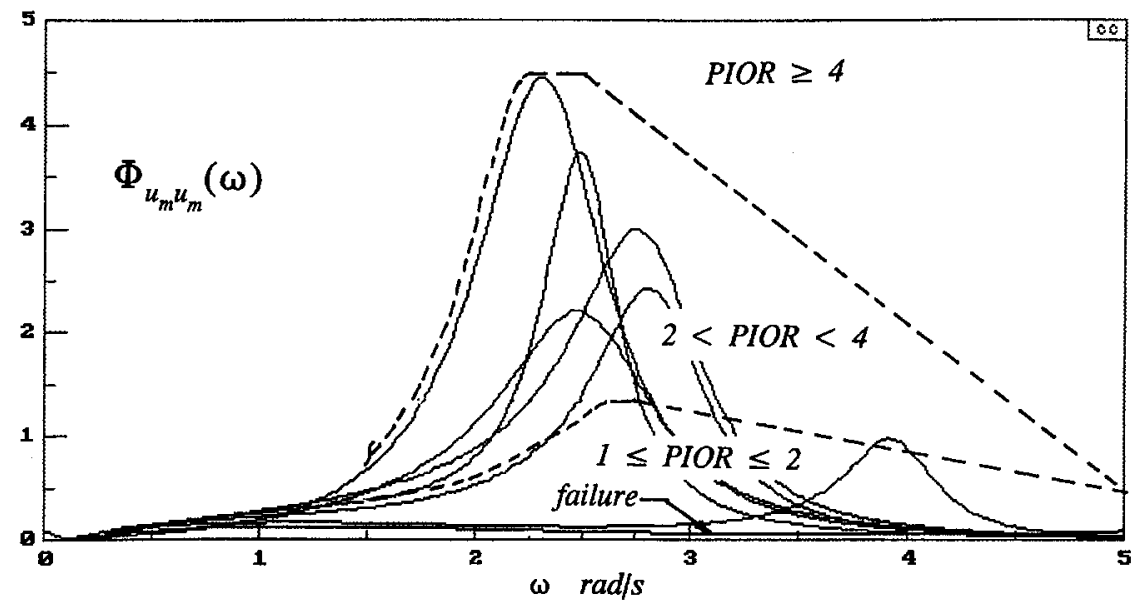

Fig. $8 \Phi_{u_{m} u_{m}}(\omega)$ for flight-test configurations receiving $2<$ PIOR $<4$.

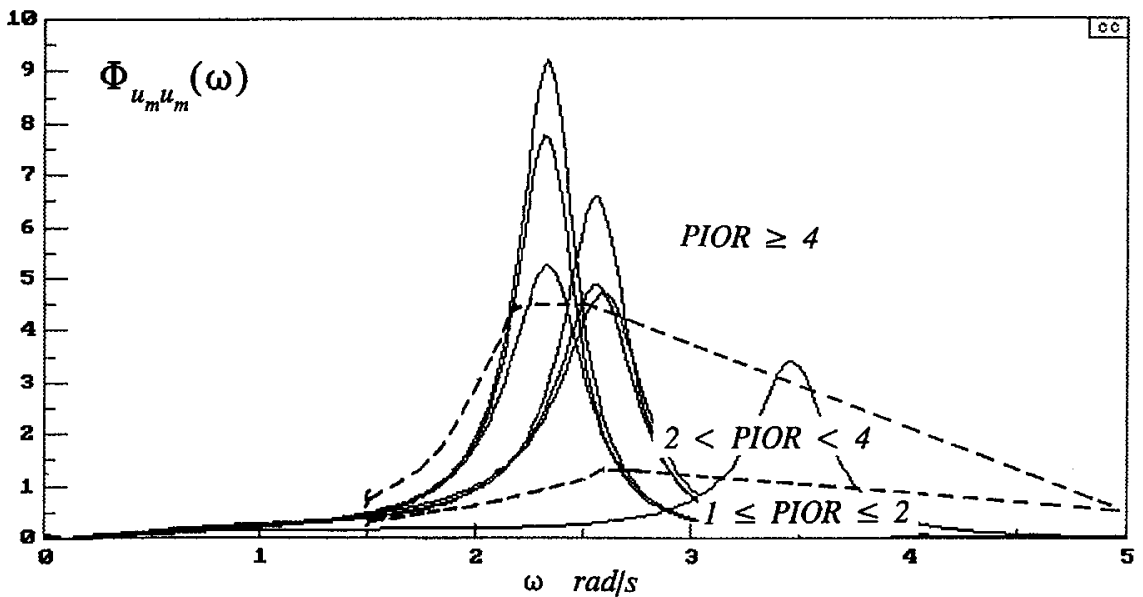

Fig. $9 \Phi_{u_{m} u_{m}}(\omega)$ for flight-test configurations receiving PIOR $\geq 4$.

PIOR $=4$, and flight-test-time histories from Ref. 17 clearly indicate APC/PIOs. A root locus analysis indicated that the frequency of oscillation will be $3.26 \mathrm{rad} / \mathrm{s}$; an APC/PIO of $3.3 \mathrm{rad} / \mathrm{s}$ occurred in flight tests. ${ }^{17}$ The closeness of this agreement is probably fortuitous. Although APC/PIO events are oscillatory, a precise frequency definition is often difficult. Indeed, in examining the time histories of single APC/PIO events, different frequencies can be detected. In research flight tests in which APC/PIO events occur with a single configuration, it is possible to see different frequencies for different pilots. For this reason, no detailed comparison of APC/PIO frequencies between model and experiment will be attempted here. In addition, and in terms of the model of Fig. 1, it is possible for the pilot to switch back to position tracking (with proprioceptive feedback) in an APC/PIO event. The fact that an APC/PIO was initiated, however, suggests that even position tracking may be oscillatory in nature. This means that the actual APC/PIO frequency(s) may also reflect the dominant frequency in $\Phi_{u_{m} u_{m}}(\omega)$. Consider configuration H3-12 (Ref. 16). There a relatively low-frequency APC/PIO of $2.2 \mathrm{rad} / \mathrm{s}$ was recorded. With switches $S_{1}$ and $S_{2}$ in the up position, a root locus analysis with the model of Fig. 1 indicated an APC/PIO frequency of $3.1 \mathrm{rad} / \mathrm{s}$, a considerably higher value than that recorded in flight tests. However, $\Phi_{u_{m} u_{m}}(\omega)$ for this case, shown 


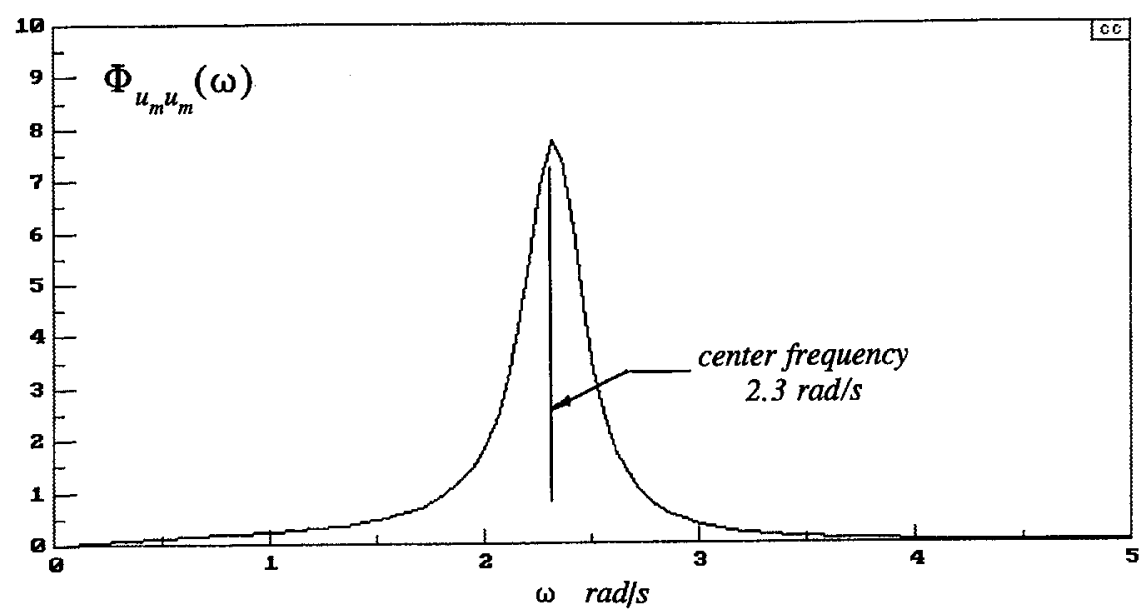

Fig. $10 \Phi_{u_{m} u_{m}}(\omega)$ for configuration H3-12 from Ref. 16.

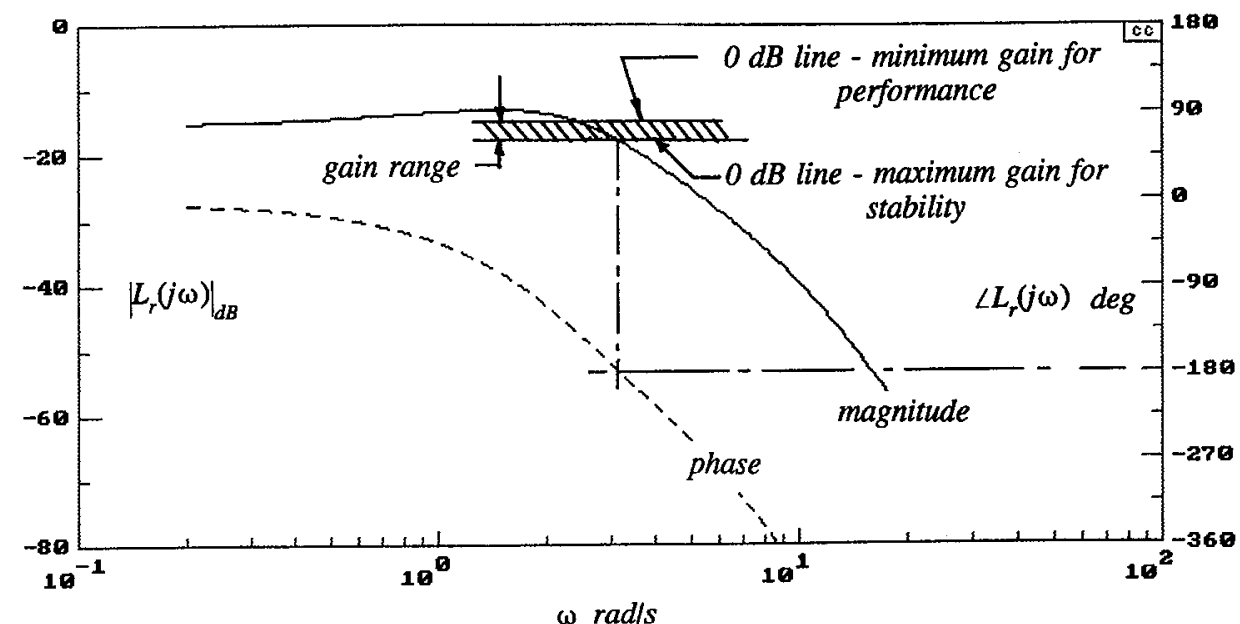

Fig. 11 Loop transmission $L_{r}(j \omega)$ for a pilot-vehicle analysis of configuration H2-5 from Ref. 16.

in Fig. 10, indicates a narrow band $u_{m}(t)$ [and, by inference, $\dot{m}(t)$ ]. This frequency band is centered at $2.3 \mathrm{rad} / \mathrm{s}$, much closer to the value obtained from flight tests. In this light, a range of possible APC/PIO frequencies may be a better prediction than a single frequency resulting from the rate-tracking model. The low frequency in this range would be that for $\left.\Phi_{u_{m} u_{m}}(\omega)\right|_{\max }$, and the high frequency would be that obtained from the root locus analysis.

Note that the range of $K_{\dot{e}}$ that the pilot can adopt during rate tracking is quite limited. This is demonstrated in Fig. 11, which shows $L_{r}(j \omega)$ as defined in Eq. (10) for the model of Fig. 1. The vehicle configuration is H2-5 (Ref. 16). Shown on the magnitude plot is the probable range of gains that the pilot could adopt, with the minimum gain corresponding to some minimum level of acceptable rate-tracking performance and the maximum gain corresponding to neutral stability. This diagram suggests why, once initiated, an APC/PIO event is not easily arrested.

\section{Inceptor Applied Force-Output Rate Phasing}

Another measure of model validity in describing APC/PIO events is the phasing between the vehicle response variable (or its rate) being controlled and the inceptor force being applied by the pilot during the APC/PIO event. Again returning to configuration $\mathrm{H} 2$ 5 (Ref. 16), Fig. 12a shows an APC/PIO event that occurred in a flight test. Note the phasing between stick force and response rate (pitch rate). When the pitch rate is experiencing an axis crossing, the control force has just passed its maximum value and is beginning to decrease in magnitude. Figure $12 \mathrm{~b}$ shows the corresponding pair of time histories from the model of Fig. 1. Note the similar phasing between pitch rate and control force. The variables in Fig. 12b have been scaled so as to produce nearly equal amplitudes. Of course, the linear, noise-free model results are much smoother than those occurring in the flight test. The APC/PIO frequency resulting from the model-based root locus analysis was $3.16 \mathrm{rad} / \mathrm{s}$. The flight-test oscillations appeared to be gradually increasing in frequency. At the end of the event, the frequency was approximately $3.1 \mathrm{rad} / \mathrm{s}$.

\section{Application to Nonlinear Vehicle Dynamics}

A convenient categorization of APC/PIO encounters has been suggested that includes three categories. ${ }^{2}$ Category I describes events with essentially linear vehicle dynamics and pilot behavior. Category II describes events in which fundamental nonlinearities come into play, chiefly, those associated with the actuators. Category III describes events that fundamentally depend on nonlinear transitions in either the effective vehicle dynamics or the pilot's behavioral dynamics. The model-based theory that has been described herein addresses only Category I events. Many of the APC/PIO encounters of modern FBW aircraft involve rate limiting in the actuators, a factor that has not been included in the analysis described here. Thus, exonerating a vehicle from APC/PIO using the techniques described here may be premature, unless one can ensure that actuator saturation, particularly rate saturation, will not produce an APC/PIO.

Extending the theory that has been described here to the case of actuator saturation should be straightforward. This is because the fundamental metric that has been used in determining APC/PIO susceptibility is simply the PSD of a signal that is easily accessible in a non-real-time simulation of the pilot-vehicle system. In addition, calculation of the HQSF with nonlinear vehicle dynamics can also be accomplished using existing techniques for determining the Laplace transforms of the input-output pairs of nonlinear systems, e.g., the technique used in nonlinear quantitative feedback theory. ${ }^{19}$ Research in this area is underway. 


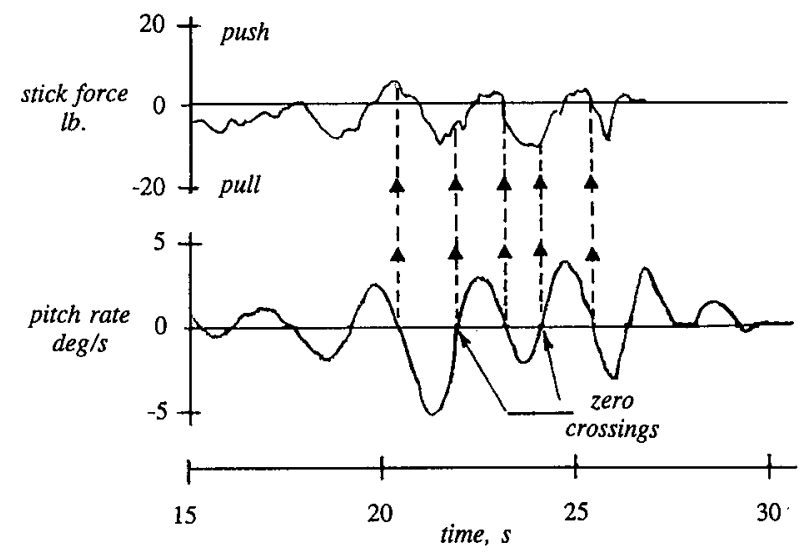

a) Flight test

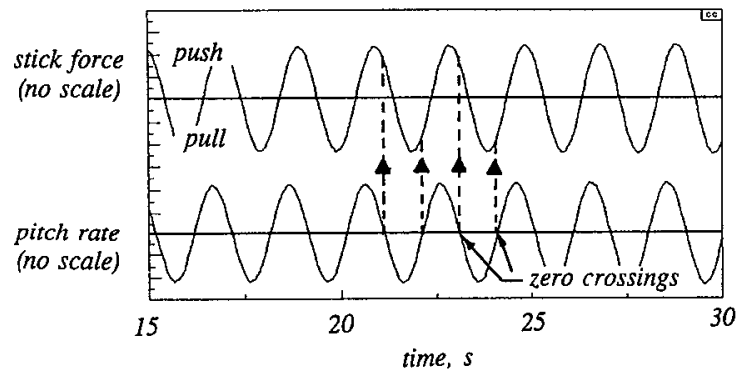

b) Pilot-vehicle analysis

Fig. 12 Comparison of phasing of applied control forces and vehicle output rate for configuration H2-5 from Ref. 16.

\section{Limitation and Caveat}

The primary limitation of the pilot-vehicle analysis that has been discussed is its inability to reproduce the changes in handling qualities and APC/PIO susceptibility that accompany changes in control sensitivity. Of course, this limitation is common to most, if not all, pilot-model-based analysis procedures. One must simply rely on experimental results to provide guidance regarding this important parameter.

The handling qualities and APC/PIO boundaries that have been derived resulted from the application of a very specific model of the human pilot, with very specific fixed parameter values and formal procedures for obtaining pilot compensation elements, e.g., $Y_{\mathrm{PF}}$ in Fig. 1. Changes in any part of this analysis will affect the boundaries that have been derived and should be avoided if one wishes to use the proposed methodology in predictive fashion.

\section{Conclusions}

Based on the research that has been described, the following conclusions can be drawn.

1) A unified theory for aircraft handling qualities and APC/PIOs is possible. The theory is based on a revised structural model of the human pilot and the central importance of a proprioceptively derived signal in that model.

2) Using a well-defined pilot-vehicle analysis technique and flight-test results, it was possible to categorize the following: i) handling quality levels using a handling qualities sensitivity function, easily derived from the pilot model; and ii) PIOR levels using the PSD of a signal easily derived from the pilot model.

3) A theory for describing pilot behavior in initiating and sustaining an APC/PIO event can be hypothesized, consistent with the feedback topology of the structural model. The theory postulates that, in initiating and sustaining an APC/PIO, the pilot regresses to a type of tracking behavior in which error rate is controlled and in which no proprioceptive feedback (and appropriate plant compensation) is used. This behavior is considered to be unwanted, inadvertent, and atypical.

4) The theory and pilot-vehicle analysis technique appear to be extendable to the study of APC/PIO events involving nonlinear vehicle dynamics such as actuator saturation.

\section{Acknowledgments}

This research was supported by NASA Langley Research Center under Grant NAG1-1744. Barton Bacon was the Contract Technical Manager.

\section{References}

${ }^{1}$ Smith, R. H., "A Theory for Longitudinal Short-Period Pilot Induced Oscillations," U.S. Air Force Flight Dynamics Lab., AFFDL-TR-77-57, Wright-Patterson AFB, OH, June 1977.

${ }^{2}$ McRuer, D. T., "Pilot-Induced Oscillations and Human Dynamic Behavior," NASA CR 4683, July 1995.

3"Flight Vehicle Integration Panel Workshop on Pilot Induced Oscillations," AGARD Advisory Rept. 335, Feb. 1995.

${ }^{4}$ Aviation Safety and Pilot Control-Understanding and Preventing Unfavorable Pilot-Vehicle Interactions, Rept. of the NRC Committee on the Effects of Aircraft Pilot Coupling on Flight Safety, National Academy Press, Washington, DC, 1997

${ }^{5}$ Klyde, D. H., McRuer, D. T., and Myers, T. T., "Unified Pilot-Induced Oscillation Theory, Volume I: PIO Analysis with Linear and Nonlinear Effective Vehicle Characteristics, Including Rate Limiting," U.S. Air Force Flight Dynamics Directorate, WL-TR-96-3028, Wright-Patterson AFB, OH, Dec. 1995.

6Preston, J. D., Citurs, K., Hodgkinson, J., Mitchell, D. C., Buckley, J., and Hoh, R. H., "Unified Pilot-Induced Oscillation Theory, Volume II: Pilot-Induced Oscillation Criteria Applied to Several McDonnell Douglas Aircraft," U.S. Air Force Flight Dynamics Directorate, WL-TR-96-3029, Wright-Patterson AFB, OH, Dec. 1995.

${ }^{7}$ Anderson, M. R., and Page, A. B., "Unified Pilot-Induced Oscillation Theory, Volume III: PIO Analysis Using Multivariable Methods," U.S. Air Force Flight Dynamics Directorate, WL-TR-96-3030, Wright-Patterson AFB, OH, Dec. 1995

${ }^{8}$ Bailey, R. E., and Bidlack, T. J., "Unified Pilot-Induced Oscillation Theory, Volume IV: Time-Domain Neal-Smith Criterion," U.S. Air Force Flight Dynamics Directorate, WL-TR-96-3031, Wright-Patterson AFB, OH, Dec. 1995.

${ }^{9}$ Chalk, C. R., "Excessive Roll Damping Can Cause Roll Ratchet," Journal of Guidance, Control, and Dynamics, Vol. 6, No. 3, 1983, pp. 218, 219.

${ }^{10}$ Hess, R. A., "A Model for the Human's Use of Motion Cues in Vehicular Control," Journal of Guidance, Control, and Dynamics, Vol. 13, No. 3, 1990 pp. $476-482$.

${ }^{11}$ Hess, R. A., "Analyzing Manipulator and Feel System Effects in Aircraft Flight Control," IEEE Transactions on Systems, Man, and Cybernetics, Vol. 20, No. 4, 1990, pp. 923-931.

${ }^{12}$ Hess, R. A., "Feedback Control Models-Manual Control and Tracking," Handbook of Human Factors and Ergonomics, 2nd ed., edited by G. Salvendy, Wiley, New York, 1997, pp. 1249-1294.

${ }^{13}$ Hess, R. A., "Analysis of Aircraft Attitude Control Systems Prone to Pilot-Induced Oscillations," Journal of Guidance, Control, and Dynamics, Vol. 7, No. 1, 1984, pp. 106-112

${ }^{14}$ Hess, R. A., Malsbury, T., and Atencio, A., Jr., "Flight Simulator Fidelity Assessment in a Rotorcraft Lateral Translation Maneuver," Journal of Guidance, Control, and Dynamics, Vol. 18, No. 1, 1993, pp. 79-85.

${ }^{15}$ Smith, R. E. "Effects of Control System Dynamics on Fighter Approach and Landing Handling Qualities, Vol. I," U.S. Air Force Flight Dynamics Lab., AFFDL-TR-78-122, Wright-Patterson AFB, OH, March 1978.

${ }^{16}$ Bjorkman, E. A., "Flight Test Evaluation of Techniques To Predict Longitudinal Pilot Induced Oscillations," M.S. Thesis, U.S. Air Force Inst. of Technology, AFIT/GAE/AA/86J-1, Wright-Patterson AFB, OH, Dec. 1986.

${ }^{17}$ Berthe, C. J., Chalk, C. R., and Sarrafian, S. K., "Pitch Rate Flight Control Systems in the Flared Landing Task and Design Criteria Development," NASA CR-172491, Oct. 1984.

${ }^{18}$ Weingarten, N. C., Berthe, C. J., Jr., Rynaski, E. G., and Sarrafian, S. K., "Flared Landing Approach Flying Qualities," Vols. 1 and 2, NASA CR-178188, Dec. 1986

${ }^{19}$ Horowitz, I., Quantitative Feedback Design Theory (QFT), QFT Publications, Boulder, CO, 1993, Chap. 11 
